\title{
Autonomic cardiovascular regulation in obesity
}

\author{
K Laederach-Hofmann ${ }^{1}$, L Mussgay ${ }^{2}$ and H Rúddel $^{2}$ \\ ${ }^{1}$ Unit of Psychosomatic and Psychosocial Medicine, University of Berne, Switzerland \\ ${ }^{2}$ University of Trier, Center for Psychobiological and Psychosomatic Research, Department of Behavioral Medicine, St-Franziska-Stift, Bad Kreuznach, Germany \\ (Requests for offprints should be addressed to K Laederach-Hofmann, Unit for Psychosomatic and Psychosocial Medicine, University of Berne, Inselspital, \\ CH-3010 Berne, Switzerland; Email: laederach@insel.ch)
}

\begin{abstract}
Obese persons suffer from an increased mortality risk supposedly due to cardiovascular disorders related to either continuously lowered parasympathetic or altered sympathetic activation. Our cross-sectional correlation study establishes the relationship between obesity and autonomic regulation as well as salivary cortisol levels. Three patient cohorts were sampled, covering ranges of body mass index (BMI) of 27-32 $(n=17), 33-39(n=13)$ and above $40 \mathrm{~kg} / \mathrm{m}^{2}(n=12)$, and stratified for age, sex and menopausal status. Autonomic cardiovascular regulation was assessed by use of heart rate variability and continuous blood pressure recordings. Spectral analytical calculation (discrete Fourier transformation) yields indices of sympathetic and parasympathetic activation and baroreflex sensitivity. Morning salivary cortisol was concurrently
\end{abstract}

collected. Contrary to expectation, BMI and waist/hip ratio (WHR) were inversely correlated with sympathetic activity. This was true for resting conditions $(r=-0 \cdot 48$, $P<0 \cdot 001 ; r=-0 \cdot 33, P<0 \cdot 05$ for BMI and WHR respectively) and for mental challenge $(r=-0.42, P<0.01$ for BMI). Resting baroreflex sensitivity was strongly related to the degree of obesity at rest (BMI: $r=-0 \cdot 35, P<0 \cdot 05$ ) and for mental challenge $(r=-0.53, P<0 \cdot 001)$. Salivary cortisol correlated significantly with waist circumference $(r=-0 \cdot 34, P=0 \cdot 05)$. With increasing weight, no overstimulation was found but a depression in sympathetic and parasympathetic activity together with a significant reduction in baroreflex functioning and in salivary cortisol levels. Journal of Endocrinology (2000) 164, 59-66

\section{Introduction}

Obesity causes a whole spectrum of subsequent health problems. The major ones are cardiovascular, metabolic, orthopedic, gastroenterological, pulmonary and psychosocial disorders. It is thus evident that obese persons suffer from an increased mortality risk due to cardiovascular complications (Drenick et al. 1980, Garrison et al. 1983, Kannel et al. 1984, Gordon \& Kannel 1986, Hubert et al. 1993). Chronic sympathetic overstimulation and increased catecholamine levels have been incriminated in obesity (Peterson et al. 1988, Rossi et al. 1989, Petretta et al. 1995, Piccirillo et al. 1996). However, the contribution of sympatho-adrenal activity to obesity is still an unresolved issue (Landsberg \& Young 1984, Dulloo \& Miller 1987, Bray 1991, Tuck 1992, Young \& Macdonald 1992). Some of the authors have suggested that the obese display altered reactions to stressors (Piccirillo et al. 1996). Equally unresolved is the question whether aberrations of the sympathetic system contribute to obesity, or are rather a consequence of it (Macdonald 1995).

Having used spectral analysis, Piccirillo et al. (1996) reported that obesity was associated with decreased sympathetic responsiveness. Obese subjects showed a higher presynaptic activation level as indices by plasma norepinephrine levels. At the same time postsynaptic sympathetic responsiveness was diminished. The decreased sympathetic stress reactivity was thought to play a role as a factor for higher mortality. A lower sympathetic activity was also found by Peterson et al. (1988), who reported an inverse correlation of sympathetic and parasympathetic activity with increasing body fat. Rossi et al. (1989) could also find a lower parasympathetic function but reported no differences in sympathetic functions in obese subjects. The former finding was again suggestive of a causal role of parasympathetic tone in sudden death. A decreased parasympathetic activity has equally been reported by Aronne et al. (1995). However, in contrast to other studies these authors reported an increase in sympathetic control. Zahorska-Markiewicz et al. (1993) observed an overreactivity of the sympathetic nervous system, but a depression in parasympathetic activity analogous to that of Piccirillo et al. (1996). A study by Aronne et al. (1997) assessed moderately overweight subjects with increasing or decreasing weight. They reported an increase in parasympathetic activity with weight loss and an inverse behavior of sympathetic drive. However, these results only included short-term differences in a population of rather fast weight 
changes. In a study on weight gain, Hirsch et al. (1991) described an inverse relationship of the amount of weight gain with lower parasympathetic drive. In obese women higher sympathetic and parasympathetic activity have been found by Gao et al. (1996), especially when there is a combination of upper body obesity and visceral obesity.

\section{Objectives}

Spectral analysis of heart rate and blood pressure variability has proven to be a useful means to gain insight into sympathetic and parasympathetic control of heart activity (Various 1996, Berntson et al. 1997). Several studies found a different behavior of the sympathetic and parasympathetic activity in obese patients. A decrease in parasympathetic activity has consistently been reported. However, findings of alteration in sympathetic drive are inconsistent. These differences might be interpreted as a consequence of the disparate use of spectral analysis techniques or conflicting interpretation of the various frequency spectra. Typically the design of those studies essentially relied on group comparisons between obese and non-obese subjects without taking relative weight differences into account. Beyond the well-known effects of obesity on well-being (Laederach-Hofmann et al. 1999a) a dysfunctional autonomic nervous system (ANS) may also give raise to various symptoms in obesity as has been shown in patients with diabetes mellitus (Laederach-Hofmann et al. 1999b).

Our study therefore investigates the aberrations in cardiovascular regulation in relation to the degree of obesity. We hypothesize that with increasing body mass in healthy obese subjects dysfunctions in autonomic cardiovascular regulation become more pronounced. This should be true for sympathetic and parasympathetic function, and for reduced heart rate variability.

\section{Patients and Methods}

Three patient cohorts, covering body mass indices (BMI) in the ranges $27-32 \mathrm{~kg} / \mathrm{m}^{2}(n=17$, ten female, seven male (including five postmenopausal women)), $33-39 \mathrm{~kg} / \mathrm{m}^{2}$ $(n=13$, seven female, six male (including three postmenopausal women)) and above $40 \mathrm{~kg} / \mathrm{m}^{2}(n=12$, seven females, five males (including four postmenopausal women)) were sampled. In each group, the mean age of females and males was identical. Additionally, estrogen is known to influence heart rate variability (Sato et al. 1995) and so all premenstrual women were investigated only in the first half of their cycle. The status in the menstrual cycle was evaluated by a thorough interview. Pre- and postmenopausal women were then included in the three weight cohorts in equal numbers. This was done to parallel the effects of age, gender and estrogen status in the weight cohorts and to allow the detection of even minimal influences of weight on spectral data (Ramaekers et al. 1998). In addition we included morning salivary cortisol determination. This was done because of the well-known effects of cortisol on insulin sensitivity and thereby on blood glucose and catecholamine levels (Peeke \& Chrousos 1995, Bjorntorp 1997). Spectral activity might eventually be related to changes in cortisol excretion over the measuring period (Miki et al. 1998, Rosmond et al. (1998).

All obese subjects were inpatients of the Psychosomatic Hospital St-Franziska-Stift (Bad Kreuznach, Germany) taking part in a $4-8$ week inpatient rehabilitation program. One of the aims of such an inpatient rehabilitation program is to initiate treatment in obese patients in order to produce weight reduction. Additionally, subsequent psychological conditions can be treated under hospital conditions in order to ensure compliance of patients and offer various opportunities for the treating team to influence the patients' course of disease (medical, psychological and social).

In our patients, obesity was typically accompanied by various other psychological and medical conditions, such as backache, knee pain or depressed mood (without fulfilling criteria of minor or major depression). Secondary diagnoses were randomly spread across the psychosomatic spectrum of disorders and concern mainly depressive and neurotic reactions as well as functional disorders. All medication which could presumably influence autonomic functions (especially antihypertensives, antidepressants, tranquilizers) was ruled out by the inclusion criteria. Equally, no hypertensive subjects (systolic BP $>160 \mathrm{mmHg}$, and diastolic $\mathrm{BP}>95 \mathrm{mmHg}$ ) were included in the study. The diagnosis of arterial hypertension was performed using WHO criteria (Vallée 1999).

All patients participated in the study during the first week of hospitalization. Testing took place in the psychophysiological laboratory of the St-Franziska-Stift from 0800 to $1000 \mathrm{~h}$. Subjects were informed about the nature of the recording, their tasks and the different test phases. Throughout the whole session subjects remained comfortably seated in a reclining chair. After application and adjustment of measuring devices an equilibration period of 20 min was applied (Sato et al. 1995) and thereafter baseline values were recorded for a 5 -min period. As a mental stress test the Bonn-Det reaction-time device (Langewitz et al. 1987) was used. Against a white background brief flashes of five differently colored lights (white, red, blue, green, orange) had to be continuously monitored and immediately responded to by pressing the corresponding response key. Stimulus frequency was adjusted automatically by a computer program referencing to the actual speed and correctness of performance. This procedure ensured inter-individually comparable and intra-individually constant levels of mental challenge throughout the whole recording period. 


\section{Measuring devices}

An electrocardiogram was recorded using a standard threepoint electrode array and amplified by a device (Fa. ZAK). Self-adhesive electrodes were attached to the right and left side of the chest just below the collar bones and below the rib cage on the left side of the body. For determination of $\mathrm{R}$ - peak-time (1 ms accuracy) the Einthoven lead II was used. Finger blood pressure was assessed continuously by a FINAPRES device (OHMED A, Becton Dickinson Med. Tech., GmbH, Erlangen, Germany) with pressure cuffs being attached to the left middle finger and held in proper height by an adjustable arm-rest. To measure breathing activity a strain gauge (Fa. ZAK GmbH Med. Tech., Kirchdorf, Austria) was positioned around the chest at the level of the xyphoid process. Depth of breathing was estimated by taking the S.D. of the respective breathing signals into account. For all subjects, breathing signals were recorded at the same amplifier settings, and at comparable positions. However, strain signals are not, per se, comparable between subjects. Therefore, the ratio of the S.D. of the breathing recordings between mental challenge and resting phase was calculated for each subject to describe the relative change in breathing depth between the two experimental phases.

Spectral analysis of heart rate variability was performed with the CARSPAN program (Mulder 1988). Data acquisition was performed with a sampling frequency of $512 \mathrm{~Hz}$. The program uses a discrete Fourier transformation of the succession of cardiac events. To account for the inter-dependency of power values with the respective heart rate, raw values of power spectral density were divided by the mean heart rate of the respective experimental phase. In addition, this procedure helps to minimize age and sex effects of heart rate interference on power spectra (Mulder 1988). The remainder was squared to yield a so-called squared modulation index. Frequency bands were defined as follows. A low-frequency component encompassed the range $0 \cdot 02-0 \cdot 06 \mathrm{~Hz}$. The midfrequency ranged from 0.07 to $0.14 \mathrm{~Hz}$. In other studies this frequency band is often defined as a low-frequency band (Sato et al. 1995, Berntson et al. 1997, Ramaekers et al. 1998). The high-frequency band covered fast fluctuations in the range $0 \cdot 15-0 \cdot 40 \mathrm{~Hz}$. The respiration frequency band covered the area $\pm 0.03 \mathrm{~Hz}$ around the median respiratory frequency. Total power represents fluctuations of the whole spectrum from 0.02 to $0 \cdot 40 \mathrm{~Hz}$. For later analysis mid- and high-frequency bands were considered relevant. The mid-frequency band reflects a mix of sympathetic and parasympathetic tone, whereas the high-frequency band is mainly influenced by parasympathetic activity (Various 1996, Berntson et al. 1997). Correspondingly, continuous blood pressure was subjected to spectral analysis. For the respective frequency ranges the modulus was determined on the basis of coherence values between systolic finger blood pressure amplitude and heart rate variation above $0 \cdot 50$. Mulder (1988) was able to show that the modulus within the mid-frequency band expresses the momentary gain of baroreflex sensitivity (Robbe et al. 1987, Honzikova et al. 1992). Several groups have confirmed the validity of non-invasively assessed baroreflex sensitivity compared with the more traditional tests (Ewing 1978, Various 1996) such as the phenylephrine method (Robbe et al. 1987).

A salivary cortisol profile was established on the day following testing. Subjects were asked to gather a saliva sample immediately after waking up (usually $0645 \mathrm{~h}$ ), $20 \mathrm{~min}$ later, and then again at 0730 and $0930 \mathrm{~h}$. All samples were stored in a refrigerator and were analyzed after termination of the study. The measurements were preformed by HPLC using internationally standardized procedures (Miki et al. 1998). To represent the individually secreted amount of cortisol (Peeke \& Chrousos 1995, Rosmond et al. 1998), the resulting area under the curve (AUC) of the morning values $(n=4$, from waking to $0930 \mathrm{~h}$ ) was taken and expressed as a concentration. This procedure is valid for both sexes (Pasquali et al. 1998).

\section{Data transformation and statistical analysis}

Values were calculated for resting and mental stress phase respectively. Statistical analyses were performed with a PC-based SAS package. As a consequence of our research design, correlative statistics are considered to fulfill the optimal use of variance in the data sets. In order to account for possible skewness of data, Spearman rank-order coefficients were calculated.

\section{Results}

\section{Descriptive statistics}

Basic values and patient characteristics are presented in Table 1.

Beside the expected group differences, the only significant difference concerns the age of male and female subjects $(t=2.03 ; P=0.05)$. Using the same descriptive approach, Table 2 presents blood pressure data, spectral power values and moduli for both experimental phases.

It must be noted that no significant differences in mean values of spectra between women and men were discernible. Equally, no differences were noted in spectral values of pre- and postmenopausal women. The respective values for pre- and postmenopausal women (all with age partialled out) were for systolic blood pressure 120.1 $\pm 13 \cdot 4$ and $129 \cdot 7 \pm 18.7 \mathrm{mmHg}(P=\mathrm{NS})$, for diastolic pressure $79 \cdot 8 \pm 7 \cdot 9$ and $86 \cdot 0 \pm 7 \cdot 4 \mathrm{mmHg}(P=\mathrm{NS})$, for resting heart rate mid-frequency band $1372 \pm 1994$ and $337 \pm 196(P=\mathrm{NS})$, for resting heart rate high-frequency band $2162 \pm 2341$ and $396 \pm 245(P<0 \cdot 05)$, and for the modulus $9 \cdot 6 \pm 6 \cdot 4$ and $5 \cdot 1 \pm 4 \cdot 8(P<0 \cdot 05)$. For the mental stress testing the values were for the mid-frequency band 
Table 1 Mean values of age, size and parameters reflecting obesity for the whole group, separated for males and females, and for the three weight cohorts (mean \pm S.D.)

\begin{tabular}{|c|c|c|c|c|c|c|}
\hline & \multirow{2}{*}{$\begin{array}{l}\text { Whole sample } \\
(n=42)\end{array}$} & \multicolumn{2}{|l|}{ Sex } & \multicolumn{3}{|c|}{ Weight cohort $\left(\mathrm{kg} / \mathrm{m}^{2}\right)$} \\
\hline & & Female $(n=24)$ & Male $(n=18)$ & $27-33(n=17)$ & $33-40(n=13)$ & $>40(n=12)$ \\
\hline Age (years) & $42 \cdot 7 \pm 9 \cdot 3$ & $44 \cdot 2 \pm 8 \cdot 2$ & $40 \cdot 7 \pm 10 \cdot 6$ & $45 \cdot 3 \pm 9 \cdot 1$ & $42 \cdot 6 \pm 9 \cdot 2$ & $39 \cdot 3 \pm 9 \cdot 8$ \\
\hline Weight (kg) & $112 \cdot 9 \pm 37 \cdot 3$ & $102 \cdot 6 \pm 29 \cdot 3$ & $126 \cdot 6 \pm 42 \cdot 8$ & $85 \cdot 3 \pm 11 \cdot 1$ & $110 \cdot 1 \pm 9 \cdot 3$ & $155 \cdot 0 \pm 42 \cdot 5$ \\
\hline Height (cm) & $171 \cdot 6 \pm 9 \cdot 3$ & $165 \cdot 5 \pm 6 \cdot 7$ & $179 \cdot 6 \pm 5 \cdot 5$ & $168 \cdot 3 \pm 9 \cdot 8$ & $174 \cdot 2 \pm 10 \cdot 3$ & $172 \cdot 5 \pm 7 \cdot 0$ \\
\hline BMI $\left(\mathrm{kg} / \mathrm{m}^{2}\right)$ & $38 \cdot 0 \pm 11 \cdot 2$ & $37 \cdot 1 \pm 8 \cdot 8$ & $39 \cdot 3 \pm 14 \cdot 0$ & $29 \cdot 9 \pm 1 \cdot 6$ & $36 \cdot 3 \pm 2 \cdot 3$ & $51 \cdot 6 \pm 12 \cdot 3$ \\
\hline Waist circumference $(\mathrm{cm})$ & $113 \cdot 8 \pm 21 \cdot 2$ & $106 \cdot 4 \pm 15 \cdot 6$ & $123 \cdot 3 \pm 23 \cdot 9$ & $97 \cdot 1 \pm 8 \cdot 6$ & $114 \cdot 6 \pm 5 \cdot 8$ & $130 \cdot 0 \pm 21 \cdot 7$ \\
\hline Salivary cortisol AUC $(\mu \mathrm{g} / \mathrm{dl} / 3 \mathrm{~h})$ & $24 \cdot 2 \pm 10 \cdot 9$ & $25 \cdot 3 \pm 11 \cdot 5$ & $22 \cdot 3 \pm 10 \cdot 3$ & $26 \cdot 5 \pm 13 \cdot 5$ & $25 \cdot 1 \pm 9 \cdot 0$ & $19 \cdot 1 \pm 7 \cdot 7$ \\
\hline
\end{tabular}

$514 \pm 816$ and $235 \pm 220(P=\mathrm{NS})$, for the high-frequency band $362 \pm 408$ and $121 \pm 111(P=\mathrm{NS})$, and for the modulus $4 \cdot 8 \pm 2 \cdot 5$ and $5 \cdot 1 \pm 4 \cdot 8(P=\mathrm{NS})$.

\section{Correlative statistics}

For the whole group of patients Spearman rank-order correlations were calculated between parameters reflecting obesity and indices of autonomic cardiovascular regulation and cortisol secretion. Table 3 presents the resulting coefficients for the whole sample.

Since age has known effects on autonomic cardiovascular regulation and was found to differ between male and female subjects, the influence of age was partialled out. Thereby, however, no significant changes to the former results were discernible. In order to highlight aberrations in reactivity, difference measures of autonomic regulation indices between test phases were included as well.

Mid-frequency values were negatively correlated with BMI and waist/hip ratio (WHR) and reached significance at rest and in response to mental challenge for BMI and for WHR at rest. The high-frequency band, which represents autonomic parasympathetic tone, was negatively correlated with various indices of obesity. This effect was most pronounced when subjects were mentally challenged. This picture is completed by the finding of lower baroreflex sensitivity values concurrent with increasing body mass in resting conditions and mental challenge. Aspects of reactivity to stress were covered by difference values. Here the mid-bands came up with significant negative correlations with increasing body mass (see Fig. 1).

Table 2 Mean values of blood pressure, spectral power values (squared modulation indices) for mid- and high-frequency bands, and estimates of baroreflex sensitivity at rest and when mentally challenged (Bonn-Det), as well as difference values between experimental phases separated for males and females, and for the three weight cohorts (mean \pm S.D.)

\begin{tabular}{|c|c|c|c|c|c|c|}
\hline & \multirow{2}{*}{$\begin{array}{l}\text { Whole sample } \\
(n=42)\end{array}$} & \multicolumn{2}{|l|}{ Sex } & \multicolumn{3}{|c|}{ Weight cohort $\left(\mathrm{kg} / \mathrm{m}^{2}\right)$} \\
\hline & & Female $(n=24)$ & Male $(n=18)$ & $27-33(n=17)$ & $33-40(n=13)$ & $>40(n=12)$ \\
\hline Blood pressure, systolic (mmHg) & $124 \cdot 6 \pm 14 \cdot 4$ & $123 \cdot 7 \pm 15 \cdot 9$ & $126 \cdot 1 \pm 12 \cdot 2$ & $121 \cdot 6 \pm 10 \cdot 0$ & $122 \cdot 9 \pm 12 \cdot 6$ & $130 \cdot 8 \pm 21 \cdot 2$ \\
\hline Blood pressure, diastolic $(\mathrm{mmHg})$ & $81 \cdot 5 \pm 9 \cdot 8$ & $82 \cdot 1 \pm 8 \cdot 2$ & $80 \cdot 7 \pm 12 \cdot 0$ & $80 \cdot 4 \pm 9 \cdot 1$ & $80 \cdot 4 \pm 9 \cdot 1$ & $84 \cdot 0 \pm 12 \cdot 0$ \\
\hline \multicolumn{7}{|l|}{ Rest } \\
\hline Mid-band & $948 \pm 1578$ & $978 \pm 1678$ & $911 \pm 1487$ & $957 \pm 1398$ & $1431 \pm 2268$ & $413 \pm 439$ \\
\hline High-band & $1634 \pm 3009$ & $1489 \pm 2075$ & $1819 \pm 3958$ & $2164 \pm 4169$ & $1685 \pm 2541$ & $873 \pm 1062$ \\
\hline Mid-band & $417 \pm 621$ & $405 \pm 656$ & $433 \pm 592$ & $346 \pm 328$ & $696 \pm 980$ & $210 \pm 252$ \\
\hline High-band & $254 \pm 351$ & $268 \pm 342$ & $237 \pm 371$ & $198 \pm 176$ & $438 \pm 553$ & $129 \pm 100$ \\
\hline Modulus & $4 \cdot 3 \pm 2 \cdot 7$ & $4 \cdot 4 \pm 2 \cdot 6$ & $4 \cdot 3 \pm 3 \cdot 0$ & $4 \cdot 6 \pm 2 \cdot 1$ & $5 \cdot 5 \pm 2 \cdot 8$ & $2 \cdot 7 \pm 2 \cdot 9$ \\
\hline \multicolumn{7}{|l|}{ Difference (rest - mental challenge) } \\
\hline Mid-band & $531 \pm 1218$ & $572 \pm 1077$ & $477 \pm 1409$ & $611 \pm 1128$ & $735 \pm 1769$ & $203 \pm 268$ \\
\hline High-band & $1379 \pm 2906$ & $1221 \pm 1789$ & $1582 \pm 3956$ & $1965 \pm 4129$ & $1246 \pm 2231$ & $743 \pm 1031$ \\
\hline
\end{tabular}


Table 3 Spearman rank-order correlation coefficients between parameters of obesity and indices of autonomic cardiovascular regulation and of cortisol secretion in the two experimental phases as well as for difference measures for the whole sample of patients $(n=42)$ with age partialled out

\begin{tabular}{|c|c|c|c|}
\hline & BMI & WHR & Waist $(\mathrm{cm})$ \\
\hline \multicolumn{4}{|l|}{ Rest } \\
\hline Mid-band & $-0 \cdot 48^{* * *}$ & $-0 \cdot 33^{*}$ & $-0 \cdot 44^{* *}$ \\
\hline High-band & $-0 \cdot 21$ & $-0 \cdot 34^{\star}$ & $-0 \cdot 14$ \\
\hline Modulus & $-0 \cdot 35^{*}$ & $-0 \cdot 28$ & $-0 \cdot 43^{* *}$ \\
\hline \multicolumn{4}{|l|}{ Mental challenge } \\
\hline Mid-band & $-0 \cdot 42^{* *}$ & $-0 \cdot 15$ & $-0 \cdot 29$ \\
\hline High-band & $-0 \cdot 30^{\star}$ & $-0 \cdot 18$ & $-0 \cdot 24$ \\
\hline Modulus & $-0 \cdot 53^{* * *}$ & -0.08 & $-0 \cdot 47^{* *}$ \\
\hline \multicolumn{4}{|c|}{ Difference (rest - mental challenge) } \\
\hline Mid-band & $-0 \cdot 33^{*}$ & $-0 \cdot 34^{*}$ & $-0 \cdot 23$ \\
\hline High-band & $-0 \cdot 12$ & $-0 \cdot 27$ & $-0 \cdot 10$ \\
\hline Modulus & $-0 \cdot 11$ & $-0 \cdot 34^{\star}$ & $-0 \cdot 00$ \\
\hline Cortisol secretion & $-0 \cdot 24$ & $-0 \cdot 25$ & $-0 \cdot 34^{*}$ \\
\hline
\end{tabular}

${ }^{*} P<0 \cdot 05 ;{ }^{* *} P<0 \cdot 01 ;{ }^{* * *} P<0 \cdot 001$.

Parasympathetic withdrawal in this instance was apparent, albeit not to a significant degree, and only WHR was correlated with diminished baroreflex functioning.

The amount of cortisol secretion in the morning hours was inversely correlated with increasing waist circumference $(r=-0 \cdot 34, P=<0 \cdot 05)$.

\section{Discussion}

The main goal of the study was to detect changes of autonomic cardiovascular regulation in obesity. An increase in body weight was expected to accompany higher sympathetic activation. However, on the basis of 42

$\mathrm{Ig} / \mathrm{m}^{2}$

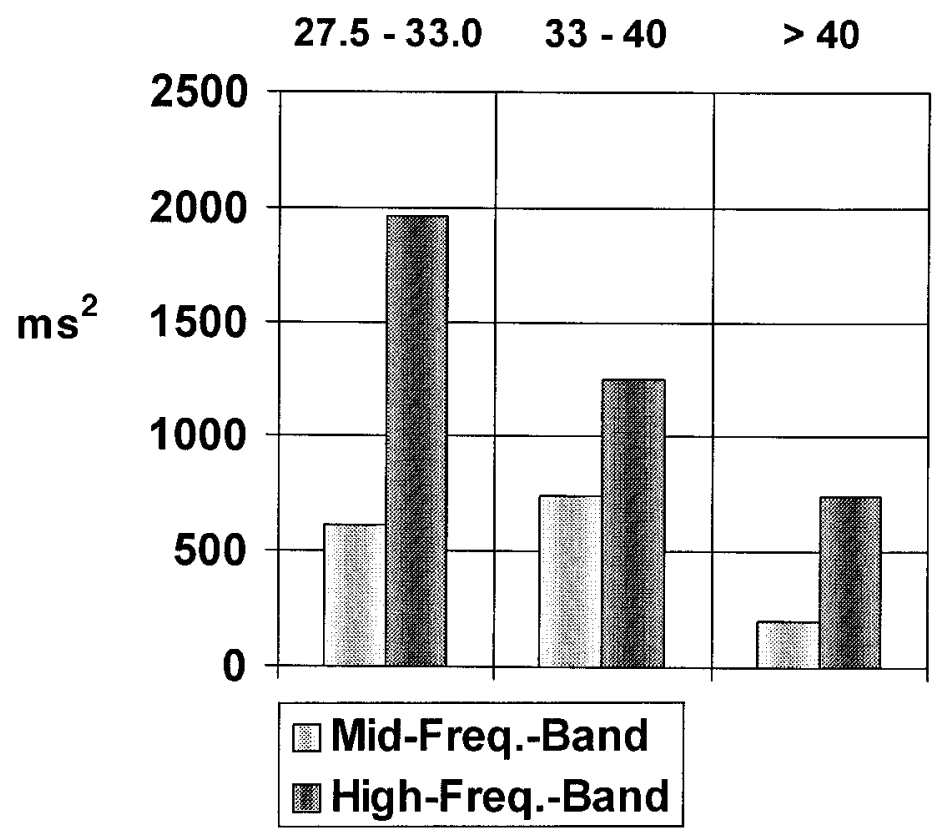

Figure 1 Difference values of mid- and high-frequency bands in obese patients of different weight classes. 
patients who have an excess BMI of $28-83 \mathrm{~kg} / \mathrm{m}^{2}$ the hypothesis of heightened sympathetic activity could not be substantiated. Nevertheless, several aspects within the emerging pattern of correlations seem worthy of note. The correlation coefficients of the mid-frequency bandreflecting a mixture of sympathetic and parasympathetic tone-are negatively correlated with BMI and WHR reaching significance at rest for WHR and additionally during mental stress for BMI. The high-frequency band, which represents autonomic parasympathetic tone, is again negatively correlated with BMI for resting conditions and with WHR for mental stress. Obviously, an increase in body weight decreases both sympathetic and parasympathetic activity. This effect is most pronounced when mentally stressed, pointing to an aggravation of parasympathetic withdrawal under stress conditions. Higher sympathetic drive, on the basis of these data, does not accompany an increase in body mass. This finding is clearly against the expectation evolving from animal studies (Scheurink et al. 1996). Whereas it might be difficult to define the different parts in mid-frequency which are associated with either sympathetic or parasympathetic activity, a lower or possibly higher sympathetic tone that is exerted might not have a detrimental effect, but might cause a deficit in parasympathetic counterregulation which is obviously particularly pronounced under stressful circumstances. This picture is completed by the finding of lower values of baroreflex sensitivity which appear with increasing body mass. Here again mental stressing seems to affect baroreflex functioning in a more pronounced fashion. Aspects of the reactivity to stress are covered by difference values. There is a significantly negative relationship between the mid-bands and an increasing BMI. Due to the way differences were calculated (resting values minus mental stress values, with resting values being higher than stress values), the resulting correlation coefficients express diminished autonomic tone to mental stress testing. The extent of the parasympathetic withdrawal is statistically not significant in this instance, and only WHR is correlated significantly with diminished baroreflex functioning. There was a negative correlation between cortisol values and waist circumference, indicating a decrease with growing abdominal obesity. Again sympathetic reactivity was found to diminish with growing body mass (see Fig. 1).

Some of the researchers who used the same methodology found similar results. Piccirillo et al. (1996), for instance, reported findings equivalent to ours. In their study obesity was found to be associated with decreased sympathetic responsiveness. Similarly, Peterson et al. (1988) reported an inverse correlation of sympathetic and parasympathetic activity with increasing percentages of body fat whereas Rossi et al. (1989) could not find any differences in sympathetic functions. Also, ZahorskaMarkiewicz et al. (1993) noted an overreactivity of the sympathetic nervous system, but depression in para- sympathetic activity - the latter in accord to our study and reported by others as well.

Another interesting finding was the decrease in parasympathetic power with growing body weight, BMI and WHR. Decreased parasympathetic activity has also been reported by Aronne et al. (1995) and Rossi et al. (1989). In a study on weight gain Hirsch et al. (1991) described an inverse relationship with the amount of weight gain which leads to lower parasympathetic power.

The novel finding of diminished baroreceptor function (i.e. resistance of the baroreceptor) with increasing weight may lead to intriguing speculations about similarities of the ANS to other organ systems that are involved in obesity. Arterial hypertension (Egan 1991) contributing to insulin resistance and the links between insulin sensitivity and fat metabolism (Dowling et al. 1995, Macor et al. 1997) closely relate to central obesity, known to be an additional risk factor in cardiovascular death (Gao et al. 1996, Scheurink et al. 1996). Gaining weight combines regularly with metabolic changes revealing adaptation processes towards 'resistance' of feedback loops involved especially in organ systems ensuring supply and utilization of energy. In addition, it has been argued that the hyperactivation of the stress axis involved in obesity is associated with a lower survival in obese patients (Peeke \& Chrousos 1995) but may over time lead to lower salivary cortisol levels (i.e. 'resistance') (Rosmond \& Bjorntorp 1998), as was found in this study. Our findings and similar results from other studies point out the importance of lowered parasympathetic activity known to exert deleterious effects on metabolism, psychosocial functioning, and survival in obese patients (Bjorntorp 1997). This applies also for normo-weight patients, especially when they suffer from diabetes mellitus (Claus et al. 1994, Laederach-Hofmann et al. 1999b).

\section{Limitations}

The different findings of activities of sympathetic or parasympathetic influences might question the reliability of the mid-frequency band as a sympathetic indicator. We agree with the Task Force report (Various 1996) and with Berntson et al. (1997), both of which showed that sympathetic contribution is mixed with mainly parasympathetic and other influences. Nevertheless it seems important to note decreased sympathetic and parasympathetic activity with growing body weight as we have reported in this study. We postulate that the results of this study are reliable due to an absence of secondary diagnoses that might have had a confounding influence. Equally, the possible influences of breathing depth have to be kept in mind. From the mathematical point of view it is obvious that depth of breathing (which was not different in our groups) can not have any influence on the spectral values (Lane et al. 1992). The breathing cycles have not been traced by a breathing volume tracer (e.g. Respitrace). 
Therefore an approximation was calculated including the S.D. of breathing frequency. The respective values in S.D. were $+21 \cdot 3$ for resting and $+22 \cdot 4$ for mental stress phases. The ratio between resting and mental stress testing phases in S.D. can be taken as a representation of alterations in the breathing depth within each subject as well as between subjects. The calculated Spearman correlations showed no significance even when the breathing frequency changed $(26.2 \pm 6.7$ in resting and $34.7 \pm 7.9$ in mental stress testing). However, in several disease states, especially when the breathing pattern is clearly abnormal (e.g. as in sleep apnea syndrome) an influence of depth of breathing on spectral values (mainly on mid-band frequency) has been reported by Khoo et al. (1999). In our patients, there has not been traced any abnormal breathing pattern be this in respect to breathing depth or frequency. Therefore we assume that our differences found between the three weight cohorts must be a specific finding related to obesity and its physiological alterations.

In conclusion the important alterations of ANS function depending on weight are a promising avenue to conduct long-term studies on weight loss and weight gain.

\section{Acknowledgements}

K L-H received a Fellowship at the Center for Psychobiological and Psychosomatic Research, University of Trier, Germany.

\section{References}

Aronne L, Mackintosh R, Rosenbaum M, Leibel R \& Hirsch J 1995 Autonomic nervous system activity in weight gain and weight loss. American Journal of Physiology 269 R222-R225.

Aronne L, Machintosh R, Rosenbaum M, Leibel R \& Hirsch J 1997 Cardiac autonomic nervous system activity in obese and never-obese young men. Obesity Research 5 354-359.

Berntson G, Bigger JJ, Eckberg D, Grossman P, Kaufmann P, Malik M, Nagarja H, Porges S, Saul J, Stone P \& Von der Molen M 1997 Heart rate variability: origins, methods, and interpretative caveats. Psychophysiology 34 623-648.

Bjorntorp P 1997 Body fat distribution, insulin resistance, and metabolic diseases. Nutrition 13 795-803.

Bray G 1991 Obesity, a disorder of nutrient partitioning: the MONA-LISA hypothesis. Journal of Nutrition 121 1146-1162.

Claus D, Feistel H, Brunholzl C, Platsch G, Neundorfer B \& Wolf F 1994 Investigation of parasympathetic and sympathetic cardiac innervation in diabetic neuropathy: heart rate variation versus meta-iodo-benzylguanidine measured by single photon emission computed tomography. Clinical Autonomic Research 4 117-123.

Dowling HJ, Fried SK \& Pi-Sunyer FX 1995 Insulin resistance in adipocytes of obese women: effects of body fat distribution and race. Metabolism 44 987-995.

Drenick E, Bale G \& Seltzer F 1980 Excessive mortality and causes of death in morbidly obese men. Journal of the American Medical Association 243 443-445.

Dulloo A \& Miller D 1987 Obesity: a disorder of the sympathetic nervous system. World Review of Nutrition and Dieting 50 1-56.
Egan BM 1991 Neurohumoral, hemodynamic and microvascular changes as mechanisms of insulin resistance in hypertension: a provocative but partial picture. International Journal of Obesity 15 (Suppl 2) 133-139.

Ewing D 1978 Cardiovascular reflexes and autonomic neuropathy. Clinical Science 55 321-327.

Gao Y, Lovejoy J, Sparti A, Bray G, Keys L \& Partington C 1996 Autonomic activity assessed by heart rate spectral analysis varies with fat distribution in obese women. Obesity Research 4 55-63.

Garrison R, Feinleib M, Castelli W \& McNamara P 1983 Cigarette smoking as a confounder of the relationship between relative weight and long-term mortality. The Framingham Heart Study. Journal of the American Medical Association 249 2199-2203.

Gordon T \& Kannel W 1986 Multiple risk functions for predicting coronary heart disease: the concept, accuracy, and application. American Heart Journal 103 1031-1039.

Hirsch J, Leibel R, Mackintosh R \& Aguirre A 1991 Heart rate variability as a measure of autonomic function during weight change in humans. Regulatory Integrative and Comprehensive Physiology 30 R1418-R1423.

Honzikova N, Fiser B \& Honzik J 1992 Noninvasive determination of baroreflex sensitivity in man by means of spectral analysis. Physiology Research 41 31-37.

Hubert HB, Feinleib M, McNamara PM \& Castelli WP 1993 Obesity as an independent risk factor for cardiovascular disease: a 26-year follow-up of participants in the Framingham Heart Study. Circulation 67 968-977.

Kannel WB, McGee DL \& Schatzkin A 1984 An epidemiological perspective of sudden death: 26-year follow-up in the Framingham Study. Drugs 28 (Suppl 1) 1-16.

Khoo M, Kim T \& Berry R 1999 Spectral indices of cardiac autonomic function in obstructive sleep apnea. Sleep 22 443-451.

Laederach-Hofmann K, Mussgay L, Schill H \& Rüddel H 1998 Gefahr der Fehleinschätzung von Körperbeschwerden bei Patienten mit Diabetes mellitus ohne pathologische klinische Befunde: Ein Positionspapier. Psychotherapie, Psychosomatik und Medizinische Psychologie (In Press).

Laederach-Hofmann K, Graf C, Horber F, Michel R, Lederer S, Lippuner K \& Schneider M 1999a Imipramine reduces frequency, number of binge eating episodes, and body weight in a subgroup of severely obese patients with binges. Results of a double-blind, randomized, placebo-controlled trial of eight weeks duration. International Journal of Eating Disorders 26 231-244.

Laederach-Hofmann K, Mussgay L, Winter A, Klinkenberg N \& Rüddel H 1999 b Early autonomic dysfunction in patients with diabetes mellitus assessed by spectral analysis of heart rate and blood pressure variability. Clinical Physiology 19 97-106.

Landsberg L \& Young J 1984 The role of the sympathoadrenal system in modulating energy expenditure. Clinics in Endocrinology and Metabolism 13 475-499.

Lane J, Adcock R \& Burnett R 1992 Respiratory sinus arrhythmia and cardiovascular responses to stress. Psychophysiology 29 461-470.

Langewitz W, Rüddel H \& Von Eiff AW 1987 Influence of perceived level of stress upon ambulatory blood pressure, heart rate, and respiratory frequency. Journal of Clinical Hypertension 3 743-748.

Macdonald I 1995 Advances of our understanding of the role of the sympathetic nervous system in obesity. International Journal of Obesity 19 (Suppl 7) S2-S7.

Macor C, Ruggeri A, Mazzonetto P, Federspil G, Cobelli C \& Vettor R 1997 Visceral adipose tissue impairs insulin secretion and insulin sensitivity but not energy expenditure in obesity. Metabolism 46 123-129.

Miki K, Kawamorita K, Araga Y, Musha T \& Sudo A 1998 Urinary and salivary stress hormone levels while performing arithmetic calculation in a noisy environment. Industrial Health 36 66-69.

Mulder L 1988 Assessment of Cardiovascular reactivity by Means of Spectral Analysis. Groningen: Universiteitsdrukkerij R U G. 
Pasquali R, Biscotti D, Spinucci G, Vicennati V, Genazzani A, Sgarbi L \& Casimirri F 1998 Pulsatile secretion of ACTH and cortisol in premenopausal women: effect of obesity and body fat distribution. Clinical Endocrinology 48 603-612.

Peeke PM \& Chrousos GP 1995 Hypercortisolism and obesity. Annals of the New York Academy of Sciences 771 665-676.

Peterson H, Rothschild M, Weinberg C, Fell R, McLeish K \& Pfeifer M 1988 Body fat and the activity of the autonomic nervous system. New England Journal of Medicine 318 1077-1083.

Petretta M, Bonaduce D, de Filippo E, Mureddu G, Scalfi L, Marciano F, Bianchi V, Salemme L, de Simone G \& Contaldo F 1995 Assessment of cardiac autonomic control by heart period variability in patients with early-onset familial obesity. European Journal of Clinical Investigation 25 826-832.

Piccirillo G, Vetta F, Fimognari F, Ronzoni S, Lama J, Cacciafesta M \& Marigliano V 1996 Power spectral analysis of heart rate variability in obese subjects: evidence of decreased cardiac sympathetic responsiveness. International Journal of Obesity and Related Metabolic Disorders 20 825-829.

Ramaekers D, Ector H, Aubert A, Rubens A \& Van de Werf F 1998 Heart rate variability and heart rate in healthy volunteers. Is the female autonomic nervous system cardioprotective? European Heart Journal 19 1334-1341.

Robbe HW, Mulder LJ, Ruddel H, Langewitz WA, Veldman JB \& Mulder G 1987 Assessment of baroreceptor reflex sensitivity by means of spectral analysis. Hypertension 10 538-543.

Rosmond R \& Bjorntorp P 1998 Endocrine and metabolic aberrations in men with abdominal obesity in relation to anxio-depressive infirmity (In Process Citation). Metabolism 47 1187-1193.

Rosmond R, Dallman MF \& Bjorntorp P 1998 Stress-related cortisol secretion in men: relationships with abdominal obesity and endocrine, metabolic and hemodynamic abnormalities (see comments). Journal of Clinical Endocrinology and Metabolism 83 $1853-1859$.

Rossi M, Marti G, Ricordi L, Fornasari G, Finardi G, Fratino P \& Bernardi L 1989 Cardiac autonomic dysfunction in obese subjects. Clinical Science 76 567-572.

Sato N, Miyake S, Akatsu J \& Kumashiro M 1995 Power spectral analysis of heart rate variability in healthy young women during the normal menstrual cycle. Psychosomatic Medicine 57 331-335.

Scheurink A, Balkan B, Strubbe J, Van Dijk G \& Steffens A 1996 Overfeeding, autonomic regulation and metabolic consequences. Cardiovascular Drugs and Therapy 10 (Suppl 1) 263-273.

Tuck M 1992 Obesity, the sympathetic nervous system, and essential hypertension. Hypertension 19 (Suppl 1) I67-I77.

Vallée J 1999 Sixth report of the Join National Committee on the prevention, detection, evaluation and treatment of arterial hypertension. Presse Medicale 24 853-858.

Various 1996 Heart rate variability. Standards of measurement, physiological interpretation, and clinical use. Task Force of the European Society of Cardiology and the North American Society of Pacing and Electrophysiology. European Heart Journal 17 354-381.

Young JB \& Macdonald IA 1992 Sympathoadrenal activity in human obesity: heterogeneity of findings since 1980. International Journal of Obesity and Related Metabolic Disorders 16 959-967.

Zahorska-Markiewicz B, Kuagowska E, Kucio C \& Klin M 1993 Heart rate variability in obesity. International Journal of Obesity 17 $21-23$.

Received 12 February 1999

Revised manuscript received 27 July 1999

Accepted 24 August 1999 